\title{
Locally manufactured wheelchairs in Tanzania - are users satisfied?
}

\author{
Amosun Seyi, Ndosi Aston, Buchanan Helen
}

1. Department of Health \& Rehabilitation Sciences, University of Cape Town, South Africa

2. Faculty of Rehabilitation Medicine, Kilimanjaro Christian Medical University College, Moshi, Tanzania

\begin{abstract}
Background: The government of Tanzania created opportunity for the production of wheelchairs that would be appropriate to the local needs and environment.

Objectives: The study assessed the extent to which the wheelchairs met the activity and participation needs of the users, as well as the users' level of satisfaction with the provision, repair and maintenance of these wheelchairs.

Methods: A descriptive cross-sectional analytical design was utilized to collect data through the administration of a questionnaire among 75 adult wheelchair users.

Results: Participants had used wheelchairs for an average period of 9.3 years. Most participants (61\%) had sustained spinal cord injuries, and used three-wheeler chairs (76\%). More than $90 \%$ reported that their wheelchairs positively influenced their activity and participation needs, and 85\% were satisfied with their ability to carry out daily activities. Participants expressed satisfaction with the durability of the wheelchairs $(89 \%)$, and the professional services received (71\%), but not with follow-up services $(77 \%)$. There was difference in satisfaction with features of 3-wheeler and 4-wheeler rigid chairs $(\mathrm{p}=0.030)$.

Conclusion: The wheelchairs positively impacted participants' activity and participation needs. Participants were satisfied with the features of the wheelchairs but not with follow-up services. The concerns of dissatisfied users should be addressed.
\end{abstract}

Keywords: Wheelchair, consumer satisfaction, mobility limitation, self-help devices, developing country

DOI: http://dx.doi.org/10.4314/ahs.v16i4.37

Cite as: Amosun S, Ndosi A, Buchanan H. Locally manufactured wheelchairs in Tanzania - are users satisfied? Afri Health Sci. 2016;16(4): 1174-1181. bttp://dx.doi.org/10.4314/abs.v16i4.37

\section{Introduction}

The primary objective of this preliminary study was to determine the extent of satisfaction among users of wheelchairs that were locally manufactured inTanzania, a low income country with an estimated population of over 49 million people in 2013. About $8 \%$ of the population experiences some type of activity limitations,

\section{Corresponding author:}

Amosun Seyi,

Department of Health \& Rehabilitation Sciences, University of Cape Town, South Africa Email: seyi.amosun@uct.ac.za though it is generally agreed that the information is unreliable and incomplete ${ }^{1-3}$. A review of literature suggests that non-specific disabilities were the most frequently reported health conditions, with neurology, communicable diseases, and road traffic trauma as the most frequent of the specific conditions examined. The country has however shown its national and international commitments to the rights of people with disabilities through the adoption of the national Policy on disability which focuses on improving the life situation and service delivery to people with disabilities, and the signing and ratification of the United Nations Convention on the rights of persons with disabilities ${ }^{2}$. Aldersey and Turn- 
bull1 expressed the need for the government to be socially accountable in turning the aspirations in these commitments into concrete actions. One of the recommended tools to monitor social accountability systems is through service delivery reports ${ }^{4}$.

With increasing numbers of persons with physical disabilities as a result of different contributory factors, the government of Tanzania acknowledged the non-suitability of donated wheelchairs by creating opportunity for the fabrication and production of wheelchairs that would be appropriate to the local needs and environment ${ }^{5}$. The training programme for technologists involved in manufacturing the wheelchairs was located at the Tanzania Training Centre for Orthopaedic Technologists (TATCOT), and the training aligns with the guidelines recommended by the World Health Organisation (WHO) and the International Society for Prosthetics and Orthotics (ISPO). The centre is recognised for providing the largest transregional training of its kind in Africa. Since 2003, three main types of wheelchairs have been developed and are commonly used ${ }^{6}$. These are the "3-wheeler" with a detachable rear wheel, and reportedly easier to maneuver in all kinds of terrains; the foldable "4-wheeler"; and the non-foldable " 4 -wheeler". However, wheelchair users in Tanzania still experience various challenges relating to mobility and community participation. Although libraries in some higher education institutions in the country provide services to people in wheelchairs, the services are not inclusive or universal ${ }^{7}$, raising concerns about the appropriateness of the wheelchairs. One of the resolutions of the Bangalore Wheelchair Consensus Conference related to the appropriateness of a wheelchair - "A wheelchair is appropriate when it is safe, durable and maintainable, meets the individual's needs and environmental conditions, provides proper fit and postural support based on sound biomechanical principles and can be accessed and sustained at the most economical and affordable price" ${ }^{\prime 8}$. Like other developing countries with limited resources, there is little information about the level of satisfaction among users of various assistive devices ${ }^{9-12}$. Therefore this preliminary study set out to determine the extent to which locally manufactured wheelchairs met the activity and participation needs of users, and the users' satisfaction with the features and services associated with the provision, repair and maintenance of the wheelchairs.

\section{Methods}

A descriptive, quantitative cross-sectional analytical design was utilized for this study, in which a questionnaire was administered to users of wheelchairs that were locally manufactured in Tanzania.

\section{Setting}

Three regions of Tanzania were selected for this study due to the differences exhibited in rural and urban settings, and landscapes. These were Kilimanjaro, Arusha and Dar es Salaam. According to the register of the Kilimanjaro Association of Spinal Cord Injured (KASI), there were many people using locally manufactured wheelchairs in these regions, and they received services from local wheelchair workshops. The register reflected approximately 250 users of locally manufactured wheelchairs.

\section{Study population and sampling}

All users registered with KASI (approximately 
250) made up the sampling frame for this preliminary study. The inclusion criteria were participants aged between 18 and 65 years old, had been using the wheelchair for at least three months, and had been living in the area of abode for at least six months. Users with cognitive impairment were excluded as they were considered as unable to provide accurate responses. Cognitive impairment was assessed by asking participants questions about their background and checking the accuracy of their responses. Unfortunately, this was not followed with a clinical evaluation due to limited resources. Data collection took place in 2012 and 2013, at a time of reports of floods in Dar es Salaam and drought in Arusha ${ }^{13}$. This led to the relocation of the residents of these areas, including people with disabilities using wheelchairs. It was therefore not possible to access all the KASI registered wheelchair users and invite them to take part in the study. With the stated constraints and being a preliminary study, a sample of convenience was recruited.

\section{Instruments}

One questionnaire, divided into four parts (AD), was used for data gathering. The first part (A) was a self-developed questionnaire seeking demographic information, history of disability and wheelchair use, description of landscape in area of abode, and overall satisfaction with the wheelchair. The second part (B) was an outcome measure, namely the Functioning Everyday in a Wheelchair (FEW) instrument14,15. The FEW rated the user's perspective of participation in ten environment and activity based situations. The responses to the statements used a six-point rating scale from "completely agree" to "com- pletely disagree". Part C of the questionnaire was the Quebec User Evaluation of Satisfaction with Assistive Technology (QUEST 2.0) instrument ${ }^{16}$ consisting of 12 questions related to satisfaction with wheelchair features and satisfaction with service provision. The instrument used a five point rating scale, from "not satisfied at all" to "very satisfied". Part D was made up of six additional questions as proposed by Samuelsson and Wressle ${ }^{17}$. The questions examined the influence of the wheelchair on activity and participation in working, leading an active leisure life, shopping, general mobility, and participating in sports. The responses were in a five point rating scale, from "not applicable" to "positively". The participants were then allowed to orally describe their personal experiences in using their wheelchairs. The questionnaire was translated into Swahili (the common language in Tanzania) through the forward and backward process.

\section{Data collection}

The study was approved by the Faculty of Health Sciences Human Research Ethics Committee of the University of Cape Town (HREC REF: 477/2012). Permission was also obtained from the Ethical Committee of the Kilimanjaro Christian Medical College of Tumaini University. A pilot study was first conducted with five wheelchair users to test participants' abilities to comprehend the questions in the instruments administered. No changes were made to the instruments. After obtaining informed consent from the selected participants, the questionnaire was administered by trained research assistants in locations agreed to with each participant.

For the FEW, participants were asked to respond to statements using a likert scale where "1" rep- 
resented "completely disagree", to "6" representing "completely agree". Similarly for the QUEST 2.0, participants were requested to respond to statements using the likert scale where " 1 " represented "not satisfied at all", to "5" representing "very satisfied".

\section{Data analysis}

Data was analyzed with the Statistical Package for the Social Sciences (SPSS ${ }^{\circ}$ ) software program version 20.0 (SPSS Inc. 2006). Frequencies and proportions were calculated for all categorical responses in sections A (participant profile), B (FEW), C (QUEST 2.0) and D (activity and participation). Medians, inter-quartile ranges and ranges were calculated for each item in Sections $\mathrm{B}, \mathrm{C}$ and $\mathrm{D}$. The chi-square test was conducted to determine if there were differences between gender and place of residence (rural/urban), satisfaction with functional needs, wheelchair features, and activity and participation. As numerical data was normally distributed, means and standard deviations were determined. In addition, the oral narratives of the experiences of the participants, as captured by the research assistants, were summarized.

\section{Results}

Seventy five users of the locally manufactured wheelchairs participated in the study (Table 1). The medical diagnoses of the participants were spinal cord injuries (61\%), cerebral palsy $(8 \%)$, traumatic injuries $(7 \%)$, post poliomyelitis $(5 \%)$, lower limb amputations (3\%), stroke (1\%), spina bifida (1\%), and others including congenital anomalies, muscular dystrophy, Tuberculosis of the spine, and gibbus (13\%). Prior to the use of the wheelchairs, the modes of mobility for the participants included crawling $(n=11)$, being carried $(n=15)$, and using crutches $(n=3)$. About a quarter of the participants $(n=19)$ started using the wheelchairs when they were younger than 18 years old. The 3 -wheeler chairs $(n=57)$ were used by most, with 33 of these users living in rural settings. This was followed by the 4-wheeler fold-able ( $n=15)$, ten of whom lived in rural settings. The 4-wheeler rigid chairs were used by 3 participants who all lived in urban settings. They started using the chairs at a mean age of 25.1 years ( $\mathrm{SD}=11.3$ years), and had used them for a duration of 9.3 years ( $\mathrm{SD}=6.9$ years). The average number of wheelchairs that had been used to date was two (range 1.0-7.0).

Most participants agreed that their wheelchairs met their functional needs (Table 2), except in the case of transportation where an appreciable number slightly agreed ( $\mathrm{n}=31$ mostly users of 4-wheeler rigid or 3-wheeler chairs). The majority of participants were satisfied with all items relating to the features of their wheelchairs, except with follow-up services (Table 3). There were no significant differences between male and female participants in wheelchair features $(p=0.652)$ and service delivery $(p=0.767)$. Similarly, there were no significant differences in the same parameters between participants in urban $(p=0.190)$ and rural areas $(p=0.530)$ respectively. However, there was a significant difference in the level of satisfaction with wheelchair features between users of 3 -wheeler and 4-wheeler rigid chairs $(p=0.030)$. For all aspects of activity and participation, except in sports, the use of the wheelchair positively influenced more than $90 \%$ of participants 
(Table 4). There were no significant differences between male and female participants $(p=0.808)$, and between participants in urban and rural areas $(\mathrm{p}=0.560)$.

Oral comments of the participants indicated that they perceived the wheelchairs to have improved their quality of life as they considered the wheelchairs as part of their bodies. They indicated that using the wheelchairs gave them opportunity to carry out various activities, either as a housewife, an employee, or in participating in income generating activities. The 3-wheeler was considered to be easier to maneuver in all kinds of terrain than the 4-wheeler. However, users of the 3-wheeler in rural settings indicated that the user had to be physically strong to propel the wheelchair uphill or ride on rough roads. Users of the 4-wheeler reported that their wheelchairs were not very safe and stable when used outdoors, especially in rough or hilly terrains. Participants recommended that a foldable 3-wheeler should be developed as this would contribute to ease of transportation. Participants also recommended the design of a braking system in stopping or slowing down the wheelchair when in motion.

The participants reported some challenges in acquiring the locally manufactured wheelchairs. Firstly, the location of the wheelchair workshops was considered to be far from where most of the people with disabilities lived. Instances were reported when there was no opportunity to assess prospective wheelchair user before the wheelchair is supplied. Even when such opportunity existed, the delivery of the wheelchair could take more than two months. Secondly, the cost of the locally manufactured wheelchair was considered exorbitant. The cost of one of the wheelchairs ranged from two hundred to three hundred US dollars. Majority of the participants reported that they received their wheelchairs as donations from KASI and other similar organisations.

\section{Discussion}

Eggers et $\mathrm{al}^{4}$ defined the process of providing wheelchairs to clients as wheelchair service delivery, and it involved ensuring the appropriateness of selected wheelchair. Wheelchair appropriateness is the degree to which the wheelchair meets the user's functional and health needs. Users with inappropriate wheelchairs may experience adverse consequences to their physical functioning, safety, quality of life, and vocational and economic standing. Appreciating the commitment of the government to the rights of people with disabilities in provision of services ${ }^{2}$, the primary objective of this preliminary study was todetermine the extent of satisfaction among users of locally manufactured wheelchairs, as part of the process of monitoring social accountability ${ }^{1}$.

Overall, the wheelchair users' satisfaction was relatively high. As with other developing countries $^{9-12}$, participants were satisfied with wheelchair related aspects such as effectiveness of use, safety, and participation in activities, though a small proportion expressed satisfaction with service related aspects. This suggests that the locally manufactured wheelchairs were perceived to be appropriate in meeting the needs of the users. This may not necessarily indicate that the resolution at the Bangalore Wheelchair Consensus Conference $^{8}$ has been achieved. For example, the outcome of this study has not given any indication that the locally manufactured wheelchairs provide "proper fit and postural support based on sound biomechanical principles". Appreciating the pre wheelchair forms of mobility report- 
ed by the participants, the locally manufactured wheelchairs, in spite of their limitations, could have contributed to improving the functionality of the users. This could have influenced the users' perceptions about the appropriateness of the wheelchairs in meeting their needs. Overall, the users seem to be most satisfied with the 3-wheeler because these are reportedly mechanically simpler to maneuver in all kinds of terrain ${ }^{6}$.

The authors however selected to focus attention on the aspects for which wheelchair users have expressed different levels of dissatisfaction. Understanding the reasons for disparities in the level of satisfaction in wheelchair users may not be easy because of the complex and multifaceted process of wheelchair service delivery ${ }^{11}$. Relating to satisfaction with respect to functional needs, the proportion of users who have expressed some degree of dissatisfaction in the use of personal or public transportation (41.3\%) cannot be ignored. While the users expressed overall satisfaction with the wheelchairs, the challenges encountered in use of transport may be due to a number of reasons. The commitment of government in the national policy on disability to take measures to ensure that transport facilities are accessible to people with disabilities should be reviewed as local commercial buses are still not equipped for people with disabilities to use them ${ }^{6}$. Similarly, more than $10 \%$ of the users expressed various degrees of dissatisfaction with the weight of the chairs, the ease of operation at different surface heights for different tasks, adjusting the chairs, safety and security, getting around indoors and outdoors, and effectiveness.

Wheelchair users (77.4\%) also expressed outright dissatisfaction with the provision of follow up services. The oral comments from the wheelchair users identified issues relating to accessibility and affordability. The affordability of the wheelchair in a country where the average monthly income was about fifty US dollars also requires attention. While the National policy on disability acknowledges that people with disabilities require technical aids, including wheelchairs, to enhance their functional ability, no clear provision has been made to assist in the acquisition of the technical aids ${ }^{1}$. Wheelchair workshops where faulty wheelchairs could be repaired, were often located very far away from where they were required. This possibly impacted the provision of services relating to the delivery of wheelchairs, the maintenance and repairs, as well as follow up services. With the commitment of the government to the rights of people with disabilities in provision of services ${ }^{2}$, the concerns of unsatisfied users should be addressed. Follow up studies should focus on the quality of life of the users ${ }^{12}$, and also identify if a correlation exists between the types of disabilities and the level of satisfaction of the wheelchair users.

\section{Limitations}

A major limitation in this study is in its research design, which was purely quantitative methodology. A mixed method research design would be more appropriate to evaluate the level of satisfaction of the wheelchair users. The number of wheelchair users participating in the study was limited by unexpected events in the country at the time (drought and floods) which had forced people to re-locate. This substantially affected the sample size as many potential participants could not be located. The sample size was therefore not sufficient to make generalizations about the population of locally-made wheelchair users in Tanzania. The findings should therefore be interpreted cautiously. 


\section{Conclusion}

This study provides some preliminary evidence on the levels of satisfaction among a small sample of people with disabilities who made use of wheelchairs that were locally manufactured in Tanzania. The users seem to be most satisfied with the functioning of the 3-wheeler chairs. Further exploration is needed to provide a more comprehensive picture. However, the findings of this study indicated that while people with disabilities expressed general satisfaction with the use of locally made wheelchairs, there are areas of dissatisfaction that require attention.

\section{Conflict of interest}

The authors declare that there is no conflict of interest. The authors themselves are responsible for the content and writing of this paper. They also declare that they have no financial or personal relationship that may have inappropriately influenced them in writing this article

\section{Acknowledgements}

The authors in a very special way acknowledge those who participated in the study particularly research assistants Mr. Daniel Namkesa and Mr. Albert Mushi; the statistician Mr. Gibson Kapanda for his statistical analysis advice; the initial funders of the study GIZ (InWENT); the Department of Health and Rehabilitation Sciences of the University of Cape Town for the financial support which enabled the second author to travel from Tanzania to South Africa; and the study participants who agreed to participate in the study.

\section{References}

1. Aldersey HM, Turnbull HR. The United Republic of Tanzania's National Policy on Disability: A Policy Analysis. Journal of Disability Policy Studies 2011; 22(3): 160-169.
2. Njelesani J, Couto S, Cameron D. Disability and rehabilitation in Tanzania: a review of the literature. Disability and Rehabilitation 2011; 33(2324): 2196-2207.

3. Mfinanga DA. Implication of pedestrians' stated preference of certain attributes of crosswalks. Traffic Policy 2014; 32: 156-164.

4. Eggers SL, Myaskovsky L, Burkitt KH, Tolerico M, Switzer GE, Fine MJ, Boninger ML. A preliminary model of wheelchair service delivery. Archives of Physical Medicine and Rebabilitation 2009; 90 (6): 1030-1038.

5. Journal of Rebabilitation Medicine 2013; 45(4): 385-391.

6. Winter AG. Assessment of wheelchair technology in Tanzania. International Journal for Service Learning in Engineering, 2006; 2(1): 60-77. 7. Majinge RM, Stilwell C. Library services provision for people with visual impairments and in wheelchairs in academic libraries in Tanzania. South African Journal of Libraries \& Information Sciences 2013; 79(2): 39-50.

8. Sheldon S, Jacobs NA. ISPO consensus conference on wheelchairs for developing countries: Conclusions and recommendations. Prosthetics and Orthotics International 2007; 31(2): 217-223. 9. Magnusson L, Ahlström G, Ramstrand N, Fransson EI. Malawian prosthetic and orthotic users' mobility and satisfaction with their lower limb assistive device. Journal of Rehabilitation Medicine 2013; 45(4): 385-391.

10. Magnusson L, RamstrandN, Fransson EI, Ahlström G. Mobility and satisfaction with lower-limb prostheses and orthoses among users in Sierra Leone: A cross-sectional study. Journal of Rehabilitation Medicine 2014; 46(5): 438-446.

11. Visagie S, Sheffler, E, Scheider M. Policy implementation in wheelchair service delivery in a rural South African setting. African Journal of Disability 2013; 2(1): 1-9.

12. Shore $S$, Juillerat $S$. The impact of a low cost wheelchair on the quality of life of the disabled 
in the developing world. Medical Science Monitor 2012; 18(9): CR533-542.

13. Mutanga SS, Mwiruki B. Managing vulnerability to climate extremes in Africa: Some policy insights into frequent flooding in Dar esSalaam, Tanzania. African Institute of South Africa 2013; 82: 1-9

14. Holm MB, Mills T, Schmeler M, Trefler E. Development and validation of the Functioning Everyday with a Wheelchair (FEW) outcome measures. Rehabilitation Engineering and Assistive Technology Society of North America, 2003; 26th International Conference, Atlanta, GA.
15. Mills TL, Holm MB, Schmeler M. 2007. Testretest reliability and cross validation of the Functioning Everyday With a Wheelchair Instrument. Assistive Technology 2007; 19(2): 61-77.

16. Demers L, Lambrou WR, Ska B. The Quebec User Evaluation of satisfaction with assistive technology (QUEST 2.0). Technology and Disability 2002; 14: 101-105.

17. Samuelsson K, Wressle E. User satisfaction with mobility assistive devices: An important element in the rehabilitation process. Disability and Rehabilitation 2008; 30(7): 551-558. 